\title{
Burned-out in a mixed germ cell tumor of the testis: The problem of pTO. Case report
}

\author{
Carlos Miacola, Ottavio Colamonico, Carlo Bettocchi, Vito Ricapito, Silvano Palazzo, \\ Marcello Campagna, Michele Battaglia, Pasquale Martino
}

Policlinico di Bari, Divisione Urologia Universitaria I, Bari, Italy

\begin{abstract}
Summary
Germ cell tumors constitute the majority of all testicular tumors, which are relatively rare overall and are mainly encountered in young adults and teenagers. The term 'burned-out' germ cell tumor refers to the presence of a metastatic germ cell tumor with histological regression of the primary testicular lesion. Clinical examination of the testes and scrotal sonography is the initial diagnosis of such neoplasms. We report an unusual case of a burned-out testicular tumor with metastases to retroperitoneal lymphnodes in an asymptomatic patient with right testicular hypoechoic nodule associated with multiple calcifications of the testicular parenchyma.
\end{abstract}

KEY WORDS: Burned-out tumor; Germ cell tumor; Testicular tumor.

Submitted 3 October 2014; Accepted 31 October 2014

\section{Case report}

A 36 years old, asymptomatic and azoospermic patient, with secondary infertility and a testicular ultrasound detection of multiple calcifications distributed throughout the parenchyma associated an hypoechoic area of $22 \mathrm{~mm}$ with peripheral calcifications in the right testicle (a testicular nodule was appreciable at palpation with negative tumor markers). Excision of the nodule was performed. Extemporaneous histological examination showed fibrosis, while the definitive one showed seminiferous tubules transformed into areas of sclero-hyalinosis (95\%), a micro-focal of adenomatous hyperplasia of rete testis and moderate interstitial fibrosis. The following year, the patient presented a rise of tumor markers ( $\beta$-hCG 8.14 $\mathrm{mIU} / \mathrm{ml}$ and $\alpha$-FP $125 \mathrm{IU} / \mathrm{ml}$ ) without symptoms; testicular ultrasound showed an hypoechoic nodule of the right testicular with calcification of surrounding parenchyma; left testicle was normal. We performed a right orchiectomy with histological examination negative for testicular cancer. In the following weeks, there was a a further increase of tumor markers ( $\beta$-hCG $15.79 \mathrm{mIU} / \mathrm{ml} \alpha$-FP $705 \mathrm{IU} / \mathrm{ml}$ and LDH $909 \mathrm{U} / \mathrm{l}$ ) associated with enlarged retroperitoneal lymph nodes at the CT scanning. A large tumor surrounding abdominal vessels and infiltrating the last ileal loop was unresectable at surgical exploration; a surgical biopsy reveled mixed germ cell tumor composed of yolk sac tumor, embryonal carcinoma and seminoma. In the following days the patient showed a rapid deterioration of lung function resulting in death from pulmonary embolism.

\section{Discussion}

It is important to distinguish burned-out tumor of the testis from extragonadal germ cell tumor. In the first case, orchiectomy change prognostic outcome $(1,2)$. In this case report, the early diagnosis of a burned-out tumor of the testis was made difficult by the absence of symptoms and by the late raising oh neoplastic markers; microlithiasis at testicular ultrasound seems associated with burned-out tumor (3).

The 'burned-out' phenomenon in germ cell tumors refers to a germ cell tumor in extra-gonadal tissues with spontaneous regression of an intra-gonadal tumor.

Extragonadal germ cell tumors are usually found in the retroperitoneal, supraclavicular, cervical, and axillary lymph nodes and occasionally in the lung and liver. There are two theories to explain this phenomenon. The first is spontaneous regression of a primary germ cell tumor after metastasis of the germ cell tumor. The mechanism of this regression is yet to be elucidated. Possible explanations are an immune response or ischemia caused by the neoplasm outgrowing its blood supply due to its high metabolic rate. A second hypothesis is the denovo development of a primary germ cell tumor in extragonadal tissues (4). Patients with extra-gonadal germ cell tumors with the burned out phenomenon usually complain of many vague symptoms, such as flank pain, abdominal mass, night sweating, or scrotal pain, and occasionally reveal an elevated testicular tumor marker. In our case, the patient complained of left-sided back pain without elevation of tumor markers (4).

Histological features that are helpful in establishing a diagnosis of a regressed testicular germ cell tumor include, apart from the scar formation, intratubular calcifications, lymphoplasmacytic infiltrate, hemosiderincontaining macrophages, and testicular atrophy (5). 
Figure 1.

Hypoechoic area on the right testis with multiple parenchyma calcifications.

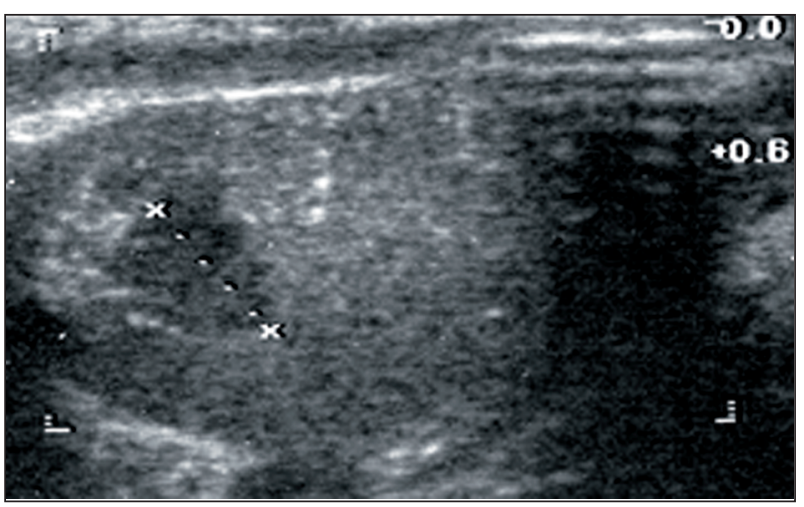

Figure 2.

Retroperitoneal lymph node package surrounds aorta and invades cava.

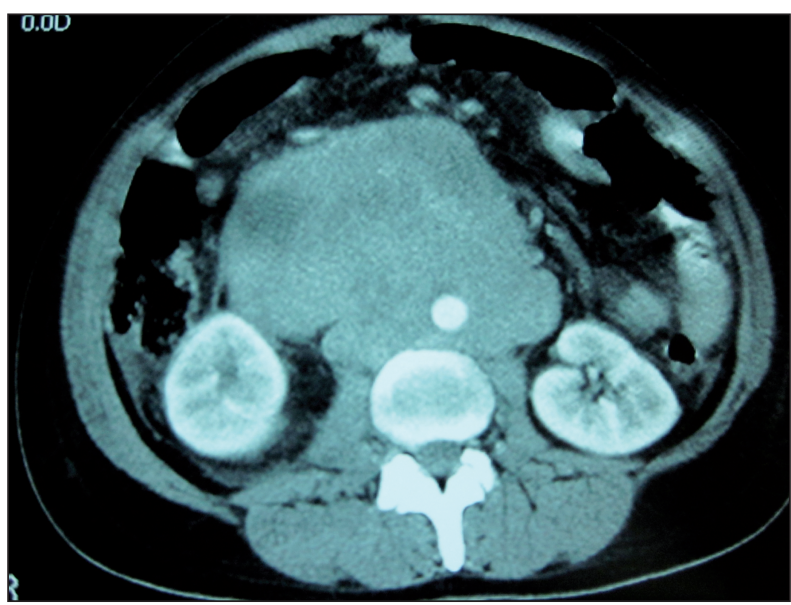

Figure 3.

Bilateral massive pulmonary embolism.

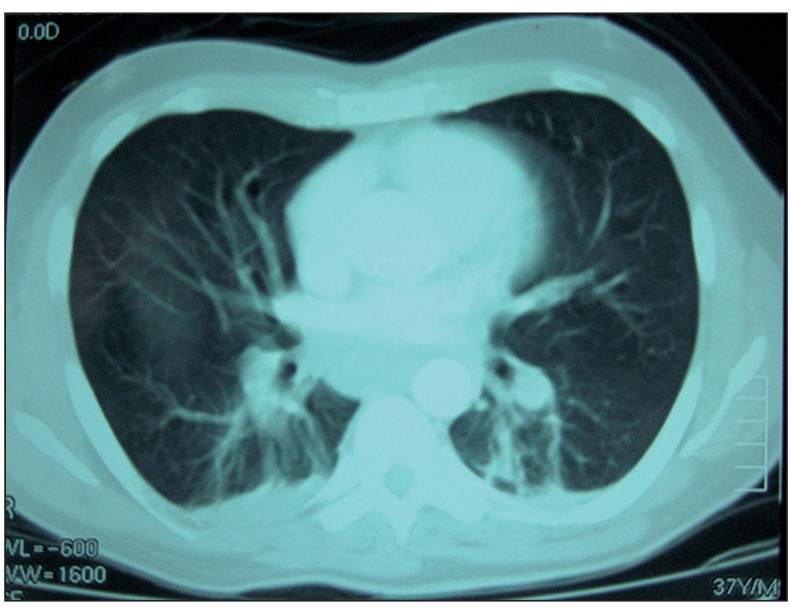

\section{Conclusion}

The management of non-palpable lesions of the testis had to provide the execution of targeted multiple testicular biopsies to exclude the presence of cancer in the testicular tumor regression context in its primary site and, above all, a careful evaluation of the retroperitoneal lymph nodes in the suspicion of pT0 that, when not diagnosed in time as in the present case, can also evolve to the exitus.

\section{REFERENCES}

1. Sahoo PK, Mandal PK, Mukhopadhyay S, Basak SN. Burned out seminomatous testicular tumor with retroperitoneal lymph node metastasis: a case report. Indian J Surg Oncol. 2013; 4:390-2.

2. Gurioli A, Oderda M, Vigna D, et al. Two cases of retroperitoneal metastasis from a completely regressed burned-out testicular cancer. Urologia. 2013; 80:74-9.

3. Sidhu PS, Muir GH. Extragonadal tumor and testicular microlithiasis: "burned-out" tumors are represented by macrocalcification. J Ultrasound Med. 2011; 30:1604-5.

4. Ha HK, Jung SG, Park SW, et al. Retroperitoneal seminoma with the 'burned out'phenomenon in the testis. Korean J Urol. 2009; 50:516-519.

5. Bär W, Hedinger C. Comparison of histologic types of primary testicular germ cell tumors with their metastases: consequences for the WHO and the British Nomenclatures? Virchows Arch A Pathol Anat Histol. 1976; 370:41-54.

\section{Correspondence}

Carlos Miacola, MD (Corresponding Author) cmiacola@hotmail.it

\section{Ottavio Colamonico, MD}

ottaviocolamonico@gmail.com

Carlo Bettocchi, MD

carlo.bettocchi@uniba.it

Vito Ricapito, MD

vito.ricapito@uniba.it

Silvano Palazzo, MD

silvano.palazzo@uniba.it

Marcello Campagna, MD marcecampagna@gmail.com

Michele Battaglia, MD

michele.battaglia@uniba.it

Pasquale Martino, MD

martino@urologia.uniba.it

Policlinico di Bari - Divisione Urologia Universitaria I

Piazza G. Cesare 11 - 70124 Bari, Italy 